\title{
Desempenho de graduandos de Odontologia no Exame Nacional de Cursos
}

\section{Análise de grupos de cursos com resultados extremos (1997-2001).}

\author{
Antonio Cesar Perri de Carvalho*, Daniel Rey de Carvalho** \\ * Professor de Cursos de Pós-Graduação da UNESP - Araçatuba e da \\ ULBRA - Canoas, consultor da UNIPLAC e do Instituto Nacional \\ de Estudos e Pesquisas Educacionais (INEP/MEC). \\ ** Mestre pela UNESP - Araçatuba, Professor da Universidade \\ Católica de Brasília e do Curso de Especialização da FOPLAC, \\ Brasília, DF.
}

\section{RESUMO}

Com o objetivo de se estabelecer relação entre os desempenhos extremos no Exame Nacional de Cursos (ENC), de 1997 a 2001, com os resultados disponibilizados pelo Instituto Nacional de Estudos e Pesquisas Educacionais (INEP/MEC) sobre algumas questões do questionário-pesquisa - preenchido pelos graduandos - sobre condições de ensino e a quantidade de graduandos, foram compostos dois grupos de cursos de Odontologia: a) os dois cursos com cinco conceitos "A” (Universidade de Brasília e Universidade Federal de Mato Grosso do Sul) e os dois cursos com quatro conceitos "A" (Universidade de São Paulo - Bauru e Universidade Estadual Paulista - São José dos Campos); b) os dois cursos com quatro conceitos "E" (Universidade Iguaçu - Rio de Janeiro e Universidade do Amazonas - Manaus).

Conclui-se que há relação entre desempenho no ENC e as condições de ensino. Os cursos com conceito "A" se diferenciam nas respostas dos graduandos nas questões sobre: equipamentos suficientes (1998 e 1999), apresentação de plano de ensino, disciplinas com conteúdo desequilibrado e atualização do docente. Esse grupo também apresenta menor quantidade de graduandos.

\section{DESCRITORES}

Ensino. Avaliação educacional.

O s cursos de Odontologia foram incluídos no Exame Nacional de Cursos, realizado pelo Instituto Nacional de Estudos e Pesquisas Educacio- nais do MEC (INEP/MEC), a partir de 1997. A prova tem sido elaborada segundo os critérios e diretrizes estabelecidos pela Comissão de Odontologia para o Exame Nacional de Cursos.

A prova constitui-se de 40 questões de múltipla escolha e 5 questões discursivas, procurando-se verificar a aquisição, pelos graduandos, das seguintes habilidades: colher, observar e interpretar dados para a construção de um diagnóstico; identificar as afecções buco-maxilo-faciais prevalentes; desenvolver raciocínio lógico e análise crítica; propor e executar planos de tratamento adequados; realizar a proservação da saúde bucal; comunicar-se com pacientes, com profissionais da saúde e com a comunidade; trabalhar em equipes interdisciplinares e atuar como agente de promoção de saúde; planejar e administrar serviços de saúde comunitária; acompanhar e incorporar inovações tecnológicas (informática, novos materiais, biotecnologia) no exercício da profissão.

Os conteúdos curriculares adotados, inicialmente, foram baseados no Currículo Mínimo para o Curso de Odontologia, aprovado pelo Conselho Federal de Educação em 1982. Assim, a prova abrangeu os seguintes conteúdos curriculares: Ciências Morfológicas, Ciências Fisiológicas, Ciências Patológicas, Ciências Sociais, Propedêutica Clínica, Clínica Odontológica, Clínica Odontopediátrica e Odontologia Social.

No dia do exame, os graduandos entregam um questionário-pesquisa, previamente preenchido, com questões sobre as suas condições sócioeconômi- 
cas e sobre as condições de ensino do curso de graduação.

Uma avaliação pioneira sobre o desempenho dos graduandos no Exame Nacional de Cursos e a relação com a avaliação das condições de oferta dos cursos (Avaliação das Condições de Oferta de Cursos de Graduação, 1998; Exame Nacional de Cursos, 1998) permitiu algumas reflexões sobre a pertinência e o valor dos itens avaliados pela Secretaria de Educação Superior do MEC (SESu), referentes aos resultados do ano de 1998 (PERRI DE CARVAlHO, 1999 e 2001a). Concluiu-se que os resultados do Exame Nacional de Cursos, efetivamente têm alguma relação com as condições de oferta levantadas na avaliação mais detalhada efetivada pelos docentes verificadores da SESu.

O objetivo deste trabalho é estabelecer relação entre os desempenhos extremos de cursos de Odontologia no Exame Nacional de Cursos, de 1997 a 2001, com os resultados de algumas questões do questionário-pesquisa - preenchido pelos graduandos -, sobre condições de ensino e a quantidade de graduandos.

\section{OBTENÇÃo de dADOS}

Foram consultados dados do questionário-pesquisa do Exame Nacional de Cursos, preenchidos pelos graduandos de Odontologia no período de 1997 a 2001, disponibilizados pelo INEP/MEC.

Considerando os desempenhos extremos dos cursos de Odontologia, foram selecionados dois grupos: a) os dois cursos com cinco conceitos "A" (Universidade de Brasília e Universidade Federal de Mato Grosso do Sul) e os dois cursos com quatro conceitos "A" (Universidade de São Paulo - Bauru e Universidade Estadual Paulista - São José dos Campos); b) os dois cursos com quatro conceitos "E" (Universidade Iguaçu - Rio de Janeiro e Universidade do Amazonas - Manaus).

No questionário-pesquisa dos citados cursos, referentes ao período de 1997 a 2001, foram coletados os resultados percentuais das seguintes questões:

1. Com que freqüência você utiliza a biblioteca de sua instituição?

2. Como você avalia a composição das disciplinas de seu curso?

3. Como você avalia o equilíbrio entre quantidade de conteúdo e carga horária das disciplinas de seu curso?

4. Ao iniciarem os trabalhos com cada disciplina, os docentes apresentam planos de ensino, contendo objetivos, metodologia, critérios de avaliação, cronograma e bibliografia?
5. Seus professores têm demonstrado domínio atualizado das disciplinas ministradas?

6. Dispunham de equipos suficientes para trabalhos em duplas, sem necessidade de subdivisão de turmas? Contavam com número adequado de pacientes matriculados para os diversos tratamentos?

Os dados sobre quantidade de graduandos foram obtidos dos Relatórios-Sínteses do Exame Nacional de Cursos, anualmente divulgados pelo INEP, com base em informações fornecidas pelas instituições.

Em seguida, obteve-se a média aritmética dos resultados das questões citadas do questionário-pesquisa e do número de graduandos, nos dois grupos de cursos, elaborando-se a representação gráfica.

\section{RESULTADOS}

$\mathrm{Na}$ análise das representações gráficas verifica-se que os cursos do grupo A (conceitos "A") se diferenciam em algumas questões.

Na questão sobre o domínio atualizado das disciplinas ministradas pelos professores, a opção " $\mathrm{D}$ - A maior parte deles demonstra" foi a mais respondida pelos graduandos (Gráfico 1).

A questão sobre o equilíbrio entre conteúdo e tempo disponível para algumas disciplinas, introduzida a partir de 1999, teve as opções relacionadas com o desequilíbrio nesta relação, como as mais assinaladas (Gráfico 2).

No tocante à questão sobre composição das disciplinas do curso, introduzida a partir de 1999, a opção "B - O currículo deveria incorporar novas disciplinas" foi a mais respondida pelos graduandos. Houve uma inversão entre os cursos dos grupos A e B entre 1999 e 2001 (Gráfico 3).

Na questão sobre apresentação do plano de ensino, a alternativa "D - A maior parte apresenta" foi a mais respondida pelos graduandos, nitidamente destacada nos cursos do grupo (Gráfico 4).

A questão sobre equipamentos suficientes, teve a alternativa "A-Sim, sempre", como a mais respondida pelos graduandos. Inicialmente com destaque para os cursos do grupo A e empatando a posição em 2001 (Gráfico 5).

A freqüência de utilização da biblioteca teve como mais respondida a alternativa " $\mathrm{E}$ - Utilizo freqüentemente", sem distinção entre os dois grupos de cursos e com forte oscilação conjunta dos dois grupos entre 1997 e 2001 (Gráfico 6).

A média da quantidade de graduandos dos cursos dos dois grupos está representada no Gráfico 7 , com predomínio de maior média para os cursos do grupo B. 


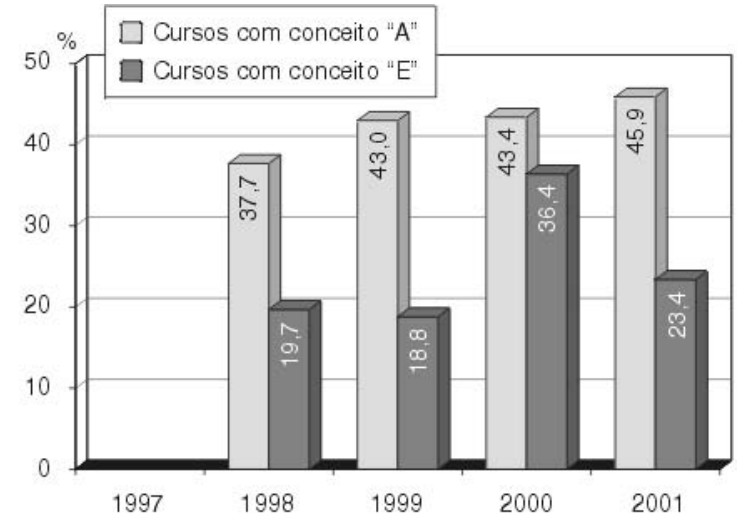

Gráfico 1 - Todos os professores demonstraram domínio atualizado das disciplinas ministradas. Fonte: INEP/MEC.

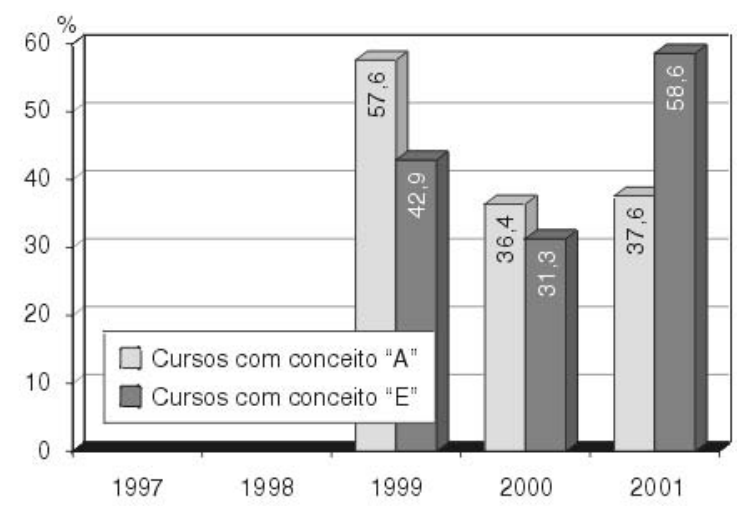

Gráfico 3 - Composição das disciplinas do curso: currículo deveria incorporar novas disciplinas. Fonte: INEP/MEC.

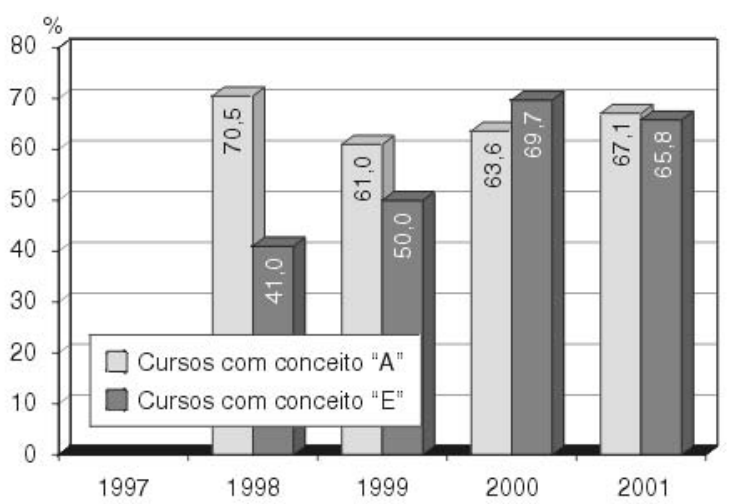

Gráfico 5 - Sempre dispunham de equipamentos suficientes para trabalho em duplas sem necessidade de subdivisão de turmas. Fonte: INEP/MEC.

\section{DIscussão}

O desempenho de graduandos no Exame Nacional de Cursos e sua relação com as condições de ensino, vêm suscitando análises nos últimos anos.

SAMPAIO (2001) comenta que até a realização do Provão, "as considerações sobre a qualidade do ensi-

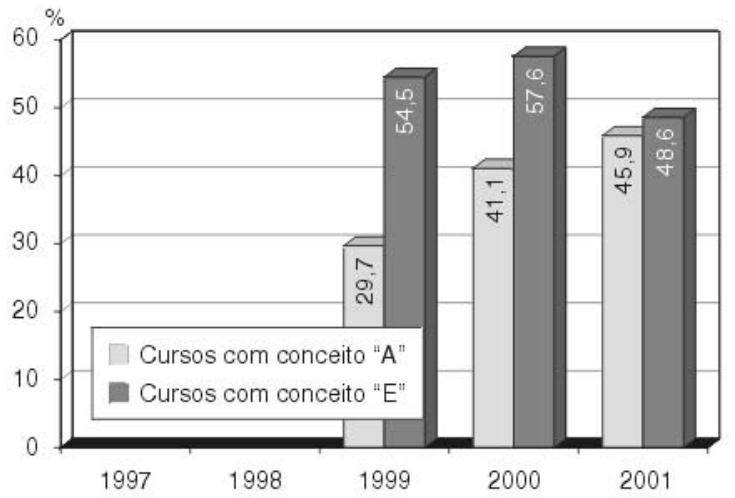

Gráfico 2 - Algumas disciplinas estão desequilibradas: muito conteúdo e pouco tempo para o seu desenvolvimento. Fonte: INEP/MEC.

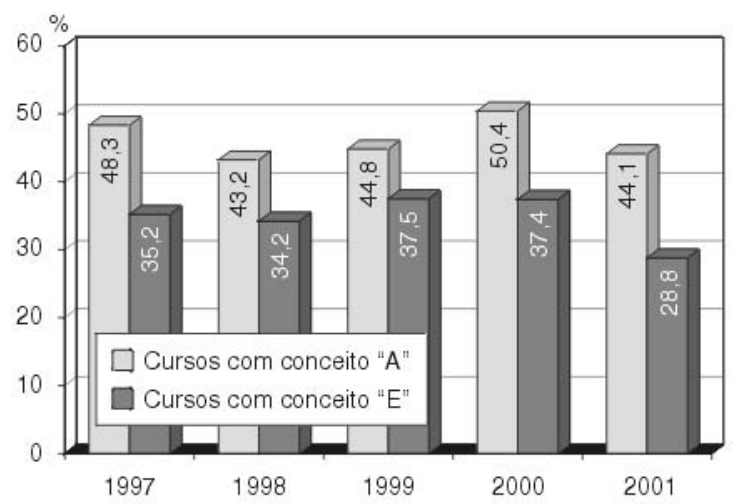

Gráfico 4 - No início de cada disciplina, todos docentes apresentam plano de ensino contendo objetivos, metodologia, critérios de avaliação, cronograma e bibliografia. Fonte: INEP/MEC.

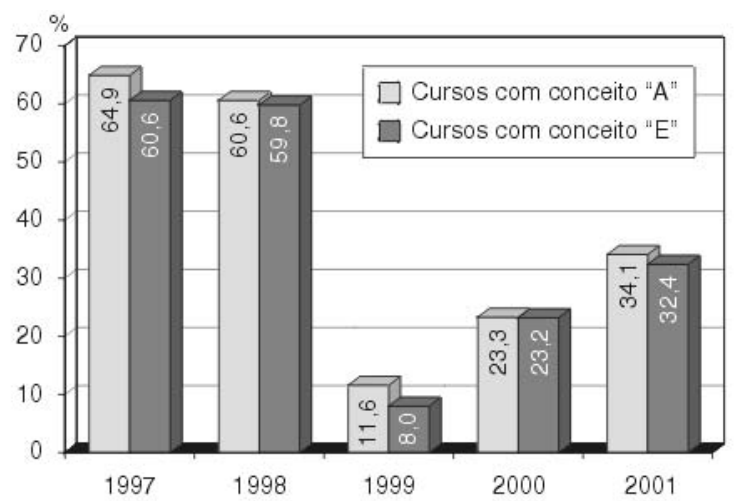

Gráfico 6 - Utilizam freqüentemente biblioteca da IES. Fonte: INEP/MEC.

no oferecido pelos estabelecimentos de ensino superior, sobretudo pelos particulares, eram inferidas a partir de indicadores indiretos como titulação e jornada de trabalho dos docentes”. Esta observação, em princípio, tem relação com os resultados que encontramos em nosso estudo que chamam atenção 


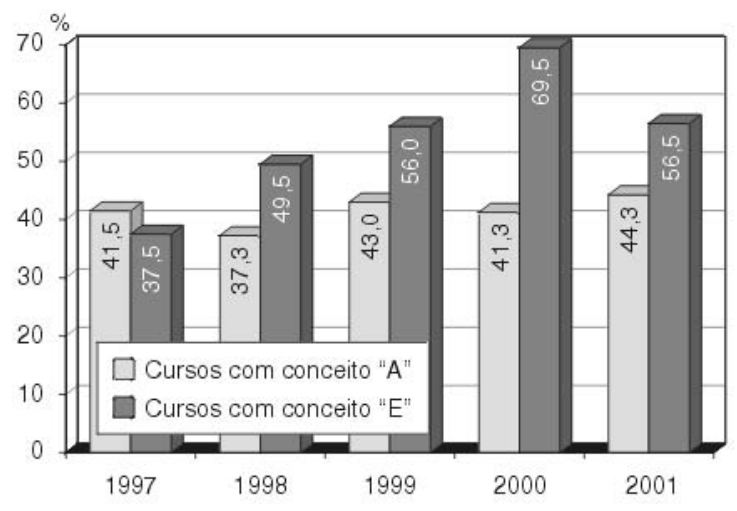

Gráfico 7 - Média da quantidade de graduandos por curso. Fonte: INEP/MEC.

para a importância do componente dedicação do docente. Os resultados dos gráficos 1 e 4 são indícios deste aspecto.

Inicialmente, nos cursos pioneiros de Odontologia, mas com reflexos posteriores, os professores dos cursos de Odontologia eram os profissionais bem sucedidos e os disponíveis para ensinarem nas faculdades. Após a fundação da USP e a instituição do regime de trabalho de dedicação integral, criado com o objetivo de impulsionar a docência e a pesquisa e, principalmente, com preparação específica e depois com os cursos de pós-graduação, ficou mais definida a atuação do professor universitário e não apenas do profissional que ministra aulas. Mesmo assim, hoje em dia, convive-se com as duas situações dentro da diversidade ensejada por instituições públicas e privadas (PERRi de CARVAlHo, 1995, 2001b).

A propósito, BALBACHEVSKY (1996) classificou e analisou os professores universitários de acordo com a estrutura da carreira institucional: contexto I - adota o critério meritocrático, que a autora considera como as instituições de melhor perfil acadêmico, como as universidades estaduais paulistas e algumas instituições federais e privadas; contexto II - reconhece e premia a dedicação à vida acadêmica, mas tem critérios alternativos para a evolução na carreira, como a maioria das instituições públicas de ensino superior (federais, estaduais e municipais) e algumas privadas; contexto III - onde há real independência entre titulação e carreira institucional, como a maioria esmagadora das instituições privadas e algumas instituições municipais e estaduais.

O mesmo raciocínio pode se relacionar com o item “organização didático-pedagógica”, pois, sem dúvida, está muito relacionado com o desempenho docente. $\mathrm{O}$ fato de que algumas instituições apresentam um rol de docentes bem qualificado em seus quadros não significa, na prática, que eles estejam atuando no dia-a-dia das atividades de graduação. Apenas aferições na rotina permitiriam a certeza de que um plano ou projeto pedagógico vem sendo efetivamente implementado. Os resultados dos gráficos 2, 3, 4 e 6 estão relacionados com a dimensão "organização didático-pedagógica”.

Em estudo sobre o Exame Nacional de Cursos em Economia, PAGNUSSAT (2001) comenta que este atualmente "é um instrumento de planejamento dos cursos, consagrou a cultura da avaliação e da imposição do aprimoramento permanente". Numa análise sobre as respostas ao questionário-pesquisa aplicado aos graduandos de Economia, entre 1999 e 2001, o autor concluiu que não houve grande alteração quanto às características dos cursos e destacou que as aulas tradicionais e não desenvolvem atividades acadêmicas além das obrigatórias. Embora o curso de Economia tenha um período de tempo menor de avaliação, provavelmente o resultado favorável do curso de Odontologia - haja vista os gráficos 2 e 4-, seja devido ao desempenho da ABENO na orientação para a elaboração de um projeto pedagógico de curso (ABENO, 1998; PERRI DE CARVALHO et al., 1998; LOMBARDO, RANALI, 1999).

A importância do processo de ensino-aprendizagem reside no fato que o processo se realiza através de um trabalho conjunto entre professor e alunos, onde o professor traça os objetivos que quer alcançar, conduzindo os alunos, fazendo-os participar em tarefas e atividades que lhes permitam construir significados cada vez mais próximos aos dos conteúdos do currículo escolar (PERRI DE CARVALHO, 2001a).

Essas considerações aparecem implícita ou explicitamente em alguns estudos sobre a prática pedagógica universitária. Em pesquisas regionais, efetivadas na alta noroeste do Estado de São Paulo e na capital paulista (PERRI DE CARVALHO, 1995; CARVAlHO $e t$ al., 1997) os formandos do interior deram maior importância ao bom entrosamento entre disciplinas e professores e, especificamente, à facilidade de contato e relacionamento com os professores. Por outro lado, entre os fatores que teriam prejudicado o andamento dos estudos, apontado pelos formandos das duas regiões citadas, foi arrolada a "má qualidade didática dos professores".

Em oportunas pesquisas com alunos de graduação das três grandes áreas de conhecimento da UNESP, GRÍGOLI (1990) observou que o "gostar de ensinar", na opinião dos alunos, comparece como qualidade fundamental num professor universitário e, inclusive, até mais importante do que "conhecer 
profundamente a disciplina que leciona". De outra pesquisa, com depoimentos de pós-graduandos na Faculdade de Odontologia da USP, MASETTO (1992) destaca alguns depoimentos dos docentes: 'Estou acostumada com o 'profissional dentista' que dá aulas e é bem diferente do 'profissional professor' que está realmente preocupado com a aprendizagem" - "Em relação às condições que facilitaram o desenvolvimento e aprendizagem do curso, colocaria em primeiro lugar o ambiente de liberdade de expressão e pensamentos que foi percebido desde o primeiro contato".

Assim, julgamos que é importante a ênfase do papel do professor de transmissor e avaliador do conhecimento, para planejador e organizador de seminários, consultor, orientador, avaliador e revisor do currículo e de temas ou unidades de aprendizagem. A atuação docente deve ser claramente atrelada a um projeto pedagógico do curso (ABENO, 1998; LOMBARDO, RANALI, 1999; PERRI DE CARVAlHO $e t$ al., 1998; PERRI DE CARVALHO, 2001a).

Nas avaliações efetivadas no ano de 1998, o item "instalações" é o que apresenta maior compatibilidade com os conceitos dos cursos no Exame Nacional de Cursos. Vale o destaque de que a infra-estrutura física e de equipamentos é um fator relevante para o desenvolvimento do curso de Odontologia. Nos resultados das verificações feitas pela SESu fica claro que há problemas importantes ligados à infra-estrutura física e às condições de funcionamento das bibliotecas, das clínicas e dos laboratórios (PERRI DE CARVAlHO, 1999). Todavia, à vista dos resultados do Gráfico 5, nosso estudo induz à interpretação de que estão ocorrendo mudanças recentes na melhoria das instalações dos cursos.

As oscilações detectadas no Gráfico 6, sobre utilização de biblioteca, sugerem que provavelmente não houve clareza e coerência por parte dos graduandos nas respostas dos anos de 1997 e 1998. Tomamos a liberdade de imaginar que os cursos não ofereçam estímulo e/ou condições adequadas para a utilização da biblioteca.

O resultado do Gráfico 7, sobre a média da quantidade de graduandos, indica que a quantidade menor de graduandos também esteja relacionada como fator que favoreça o melhor desempenho deles no Exame Nacional de Cursos.

O presente estudo permite o estabelecimento de relação entre desempenho no Exame Nacional de Cursos e as condições de ensino. Há indicações sugestivas de um processo de mudança no ensino de Odontologia. Na atualidade, as inovações pedagógi- cas, a tecnologia como subsídio à formação, aliadas ao momento de implementação das avaliações de cursos e de instituições, têm gerado novos desafios à docência universitária. Ao mesmo tempo, os avanços em todas as áreas do conhecimento contribuem para a caracterização de novo paradigma que leva ao repensar do processo ensino-aprendizagem (FERNANDES E GRILLO, 2001).

\section{CONClusóes}

Dentro das condições deste estudo é possível concluir que há relação entre desempenho no Exame Nacional de Cursos e as condições de ensino. Os cursos com conceito "A" se diferenciam nas respostas dos graduandos nas questões sobre: equipamentos suficientes (1998 e 1999), apresentação de plano de ensino, disciplinas com conteúdo desequilibrado e atualização do docente. Esse grupo também apresenta menor quantidade de graduandos por curso.

\section{ABSTRACT}

Performance of Dental undergraduate students in the National Course Exam

The relationship between extreme results in the National Course Exam, from 1997 to 2001, was studied using the publicized results of some questions of the research-questionnaire (developed by the National Institute of Studies and Researches in Education - INEP/MEC), answered by Brazilian undergraduates, about teaching conditions and number of students. Two groups of Dental courses were established: a) the two courses with five grades "A" (University of Brasília and Federal University of Mato Grosso do Sul) and the two courses with four grades "A" (University of São Paulo - Bauru and São Paulo State University - São José dos Campos); b) the two courses with four grades "E" (University of Iguaçu - Rio de Janeiro and University of Amazonas - Manaus).

It was concluded that there is a relationship between the results obtained in the National Course Exam and some teaching conditions of those Dental courses. Students in courses with "A" grades gave different answers for questions about: sufficient equipment (1998 and 1999), teaching plan presentation, disciplines with unbalanced contents and teachers' update. That group also presents less students per course.

\section{DESCRIPTORS}

Teaching. Educational measurement. 


\section{REFERÊNCIAS BIBLIOGRÁFICAS}

ABENO. Projeto pedagógico para curso de Odontologia: Anais da $33^{\underline{a}}$ Reunião da Associação Brasileira de Ensino Odontológico. Uberlândia, 1998.

BALBACHEVSKY, E. Atores e estratégias institucionais. A profissão acadêmica no Brasil. Parte I - Ensino e extensão. In: Série Documentos de Trabalho, 01/96. São Paulo : Nupes/USP, 1996.

CARVALHO, D. R., PERRI DE CARVALHO, A. C., SAMPAIO,

H. Motivações e expectativas para o curso e para o exercício da Odontologia. Rev Assoc Paul Cir Dent, v. 51, n. 4, p. 345-9. 1997.

FERNANDES, C. M. B.; GRILLO, M. Educação superior. Canoas : ULBRA, 2001. 312p.

GRÍGOLI, J. A. G. A sala de aula na universidade na visão dos seus alunos. São Paulo, 1990. Tese (Doutorado) - Pontifícia Universidade Católica.

INSTITUTO NACIONAL DE ESTUDOS E PESQUISAS EDUCACIONAIS. Exame Nacional de Cursos: relatório-síntese 1998. Brasília : MEC, 1998.

LOMBARDO, I. A.; RANALI, J. Projeto pedagógico. Manual de Orientação. Uberlândia : ABENO, 1999.

MASETTO, M. T. Aulas vivas. Tese (e prática) de Livre Docência. São Paulo : MG Editores Associados Ltda. 1992.

PAGNUSSAT, J. L. Provão de Economia 2001: sucesso e fracassos dos Cursos de Economia de Brasília. Revista de Conjuntura, v. 2, n. 8, p. 36-46, 2001.

PERRI DE CARVALHO, A. C. - Educação Ẽ saúde em Odontologia. Ensino da prática e prática do ensino. São Paulo: Editora Santos. 1995.

PERRI DE CARVALHO, A. C. Odontologia - avaliação dos cursos de graduação e conceitos obtidos no Exame Nacional de Cursos de 1998. In: Série Documentos de Trabalho, 01/99. São Paulo : Nupes/USP, 1999.

PERRI DE CARVALHO, A. C. Ensino de Odontologia em tempos da LDB. Canoas : ULBRA, 2001 (a).

PERRI DE CARVALHO, A. C. A evolução do ensino odontológico no século XX no Brasil. In: ROSENTHAL, E. A Odontologia no Brasil no século XX. Cap. IV. São Paulo : Santos, p.49-67. 2001(b).

PERRI DE CARVALHO, A. C., FERNANDES NETO, A. J., MADEIRA, M. C. Diretrizes curriculares e projeto pedagógico para curso de Odontologia. In: Série Documentos de Trabalho, 02/98. São Paulo : Nupes/USP, 1998.

SAMPAIO, H. Ensino superior no Brasil. O setor privado. São Paulo : Hucitec, 2001.

SECRETARIA DE EDUCAÇÃO SUPERIOR (SESu). Avaliação das condições de oferta de cursos de graduação: relatório-síntese 1998. Brasília : MEC, SESu, 1998. 